International Journal of Linguistics, Literature and Translation (IJLLT)

ISSN: 2617-0299 (Online); ISSN: 2708-0099 (Print)

DOI: $10.32996 / \mathrm{ijllt}$

Website: https://al-kindipublisher.com/index.php/ijllt

IJLLT

\title{
Learners' Difficulties in Self-evaluation in English Vietnamese Translation Quality Assessment
}

Tri Minh Nguyen ${ }^{1 *}$ and Trang Xuan Phuong Dao ${ }^{2}$

${ }^{1}$ Lecturer of English, Nguyen Tat Thanh University, Vietnam

${ }^{2}$ Lecturer of English, Ho Chi Minh University of Education, Vietnam

Corresponding Author: Tri Minh Nguyen, E-mail: Tringuyen.eling@gmail.com

\section{ARTICLE INFORMATION}

Received: August 06, 2020

Accepted: October 28, 2020

Volume: 3

Issue: 10

DOI: $10.32996 /$ ijllt.2020.3.10.18

\section{KEYWORDS}

translation quality assessment, difficulties, self-evaluation, culture

\section{ABSTRACT}

The field of English translation and interpretation has shown an increasing popularity in Vietnam in the context of globalization. However, translation in the target culture still has a certain number of problems in transferring the equivalence between two languages, which causes some ambiguities and misunderstandings for readers (House, 2015). Hence, translation quality assurance is significant in ensuring the degree of suitability of the texts as a post-translation process (Munday, 2016). This paper aimed to investigate the difficulties of English majors of translation and interpretation in assessing the quality of texts translated from English to Vietnamese during the learning process on an independent basis. The study employed the mixed method with 50 fourth-year students at Nguyen Tat Thanh University via questionnaires. The findings revealed that those majors had to deal with five main groups of troubles including (1) time constraints, (2) non-equivalence between English and Vietnamese, (3) insufficient cultural understandings, (4) inadequate supports from lecturers, and especially (5) the lack of official framework for translation quality assessment. This paper also proposes the fundamental for university curriculum developments from the learners' perspectives.

\section{Introduction}

\subsection{Background to the study}

Translation has been growing in popularity as a bridge among people from various lingual backgrounds to communicate with each other in the context of glocalization. In translation process, translators play a crucial role in ensuring the appropriateness of the translated products in the target language via translators' lens (Newmark, 1988; Munday, 2016). Hence, the translators need to be in possession of certain qualities to foster the effectiveness of translation process for professionalism. As a post-procedure of translation process, translation quality assessment is important as "the key to the success or failure of a cross-language communication" (Bittner, 2020, p. 17). In order to achieve the translators' attributes to be acknowledged as competence, higher education have implemented various methods in student translator training for English majors. Self-assessment as a form of formative assessment is supposed to encourage translators' reflection and autonomy to develop their skills performance (Colina, 201; House, 2015; Witter-Merithew et al., 2001).

\subsection{Statement of Problem}

In the context of Faculty of Foreign Languages, Nguyen Tat Thanh University, English majors of translation and interpretation have still dealt with certain challenges in translating the text from English to Vietnamese. As a result, the translated versions are unnatural and inappropriate in Vietnamese cultures such as bazarre word choice or foreignization of grammar. Therefore, this study aimed to investigate the difficulties faced by English majors in evaluating the quality of translation from English to Vietnamese.

\subsection{Research questions}

Based in the aforementioned problem, this study proposed two research questions:

K C AL-KINDI CENTER

$\mathbf{R}$ D FOR RESEARCH AND R D DEVELOPMENT Your gateway to world-closs research
Published by Al-KindiCenter for Research and Development. Copyright (c) the author(s). This is an open access article under CC BY license (https://creativecommons.org/licenses/by/4.0/) 
What are the difficulties of English majors in self-evaluation of translation quality at the Faculties of Foreign Languages, Nguyen Tat Thanh University?

\subsection{Significance of the study}

Translation quality assessment acts as a crucial part in the post-translation process to validate the appropriateness of the target text in the target culture. Hence, from a theoretical perspective, this study contributes to establish a fundamental in higher education of private tertiary sectors. It provides more insights into learners' challenges in assessing their translation products to ensure the quality of their career. From an empirical view, the findings from this study can be used for tertiary curriculum designs for English majors in translation and interpretation. Furthermore, lecturers can make use of this study to design the lesson with the outcome-based approach to minimize the difficulties learners encounter in professional training to enhance the teaching effectiveness for English majors.

\subsection{Scope of the study}

Due to the lack of time and financial budget, this study only focuses on exploring learners' difficulties in evaluating the quality of translation products that the participants experienced from previous formal education at the Faculty of Foreign Languages, Nguyen Tat Thanh University. However, this study did not focus on the investigation from lecturers' perspective.

\section{Literature review}

\subsection{Translation as a bridge of cultures}

The concept of translation has been proposed by various theorists from different perspectives. Newmark (1988) claims translation to be the process of "rendering the meaning of a text into another language in the way that the author intended the text" (p. 5). The meaning of the text is considered the center of translation process that has to utilize several strategies to ensure the success of meaning delivery.

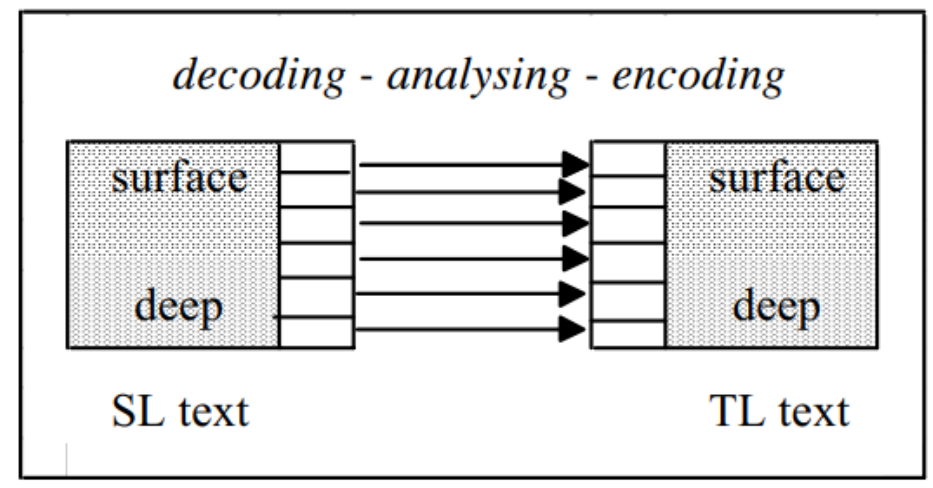

Figure 1: The Decoding-Encoding Translation Model (Katan, 2014)

According to Katan's (2014) model of translation, this process involves decoding the messages from the surface and the depth of the texture to analyze the intention of the writers from the source language texts to convey to the target language text (p. 168). It aims to explore different embedded values and meanings to conduct a successful translanguaging procedure.

However, the meaning of an entity is not always available for all the cases between two language. Hence, translation is also involved in exploring the equivalence of different translation units in the target language culture (Baker, 2018; Maitland, 2017; Nida, 2002). The lack of complete one-by-one translation between two languages has raised the issue of equivalence from various text components in translation such as sense, form, and function.

\subsection{Equivalence in translation}

The notion of equivalence in translation has spurred a notable interest among research scholars. Equivalence in translation refers to the possibility of readily appropriate expressions between two languages to represent the same entity (Munday, 2016; Nida, 1964; Pym, 2010). Translation and equivalence have a mutual relationship to shape the concept of each other in different particular translating circumstances (Kenny, 2009). The satisfaction of the complete equivalence is regularly determined by the translators who deal with the specific textual materials. 
In the rise of Computer-Assisted Translation (CAT), Pym (2007) divides translation equivalence into two types of (1) natural equivalence to focus on exploring the natural terms or chunks between the source language and the target language and (2) directional equivalence to render the source language meaning to the equivalent expressions in the target language. This approach utilizes translation tools to provide the translators with immediate versions of translation from corpus-based linguistics.

When analyzing the level of equivalence, Baker (2018) classifies equivalence from word, grammar, textuality, pragmatics, and semiotics. The author introduces the notion of implicature into translation to produce an in-depth translation products in which the message can be interpreted further than it is actually said. This type of meaning is not identified based on the single context but with the regards to the emotional and sensory effects of the writers and the readers. Translation equivalence has to deal with the illocutionary and perlocutionary acts of the original texts to transfer the same effects on the readers.

\subsection{Translation quality assessment and self-assessment}

The quality of translation products are always the ultimate goal for all translators to deal with equivalence in translation fields. Nida (1969, as cited in Munday, 2016) claims that the success of translation need to assure four main components including (1) making sense; (2) conveying the message and manner of the original; (3) having a natural and easy form of expression, and (4) producing a similar response. This idea has shed the light from reader-oriented translation approach to take the readership into account in order that the subjectivity of translators can be reduced in the translation process.

From a functional theory of translation, Newmark (1988) suggests four levels of translation that translators are supposed to achieve in the process of interlingual translation. First, the textual level deals with the grammatical structures and lexical units to transfer from the $S L$ texts to the appropriate TL texts in the semantic contexts. Second, the referential level involves the negotiation of the text and the reality of the setting. Moreover, the cohesive level aims to build the connection among components of the text and the mood that the writers imply. Finally, the level of naturalness requires translators to reproduce the SL text into an "ordinary" version in the TL culture (p.26). In other words, this level demands for the acceptability of translation products in the target setting of the texts (Colina, 2015).

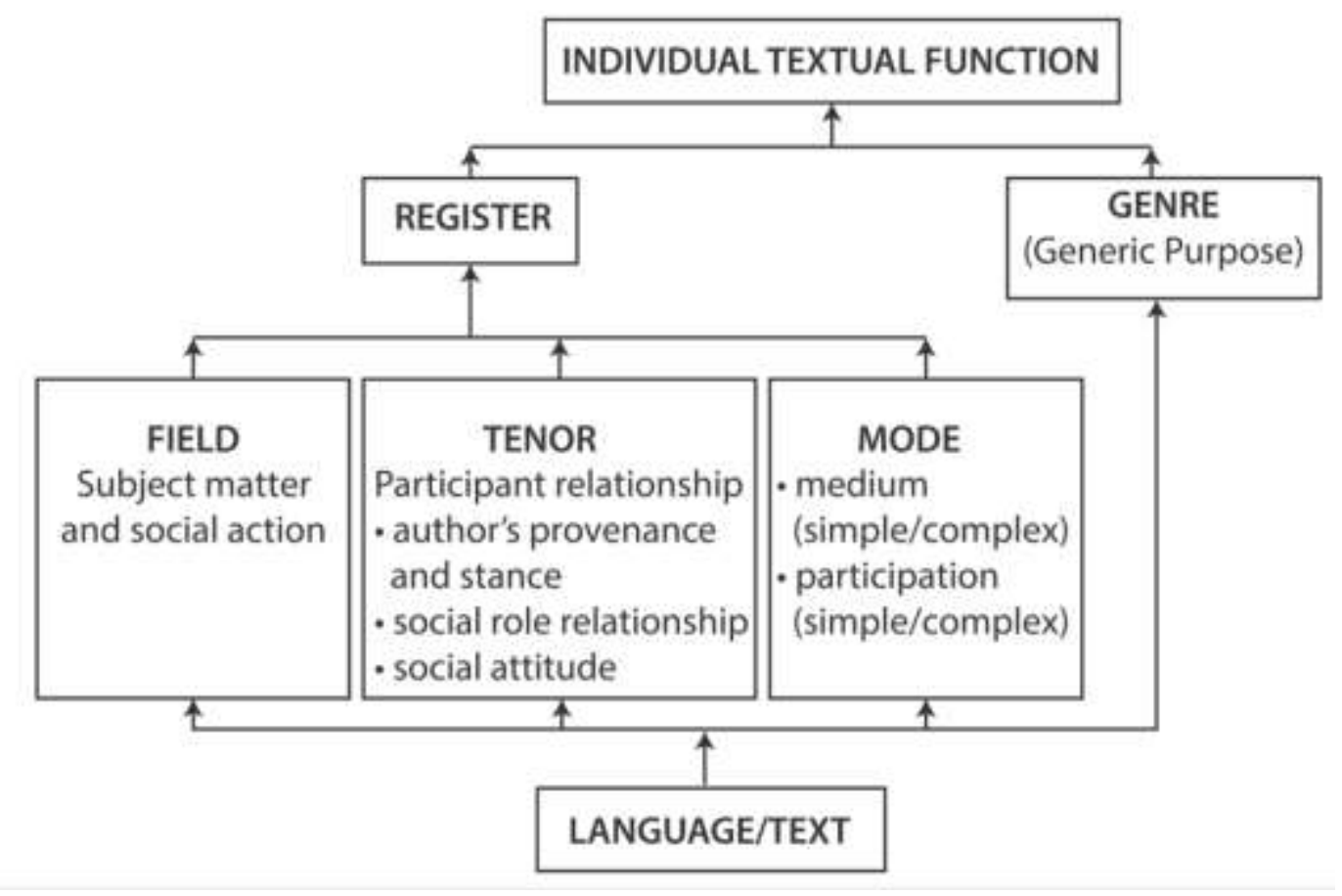

Figure 2. A scheme for analysing and comparing original and translation texts (House, 2015, p.65) 
From a linguistic-textual analysis, House (2015) proposes a scheme in Figure 2. for translator to compare the original and translation texts in the process of translation quality assessment. This scheme adopts the functional perspective of the texts to compare and contrast the register and genre of the text. The register consists of field, tenor, and mode to illustrate the correlation between the text and the context. The context plays a role in shaping the ways readers comprehend in the textual materials to figure out the intended meaning (Patridge, 2012). Furthermore, the genre is of importance to allow translators' reference to the class of text to "connect the text with the macro-context of the linguistic and cultural community" to understand the speech functions (House, 2015, p.64).

As a form of alternative assessment to produce the washback on learners, self-assessment in translation raises the learners' awareness of the objectives of the translation (Huertas-Barros, 2019). The involvement in translation as self-assessment requires student translators to identify their personal competence in terms of strengths and weaknesses. As a result, they are able to adapt and utilize several strategies to maximize their strengths and minimize the weaknesses in translation process (Lee, 2005). Additionally, self-assessment is beneficial to foster the learning autonomy for pre-service translators (Han \& Riazi, 2018; Sandrelli, 2015).

\subsection{Previous studies}

In a study by Al-mara (2016) to identify the role of self-assessment in translator education, the findings reveal that the high competent learners are those can show their awareness of the assessment criteria in translation. Another study by Lee (2016) reports that learners have difficulties in applying the criteria into the actual translation and they frequently determine the appropriateness of translation based on personal intuition.

In the context of Vietnam, a study by Nguyen \& Nguyen (2019) on the difficulties in audiovisual translation, the findings show that the problems in translation from English into Vietnamese are from the lack of equivalence, inadequacy of cultural awareness, and translators' language proficiency.

\section{Methodology}

\subsection{Setting and participants}

The study was conducted on forty four fourth year students of English majors in translation and interpretation at Faculty of Foreign Languages, Nguyen Tat Thanh University. The participants language proficiency ranges from intermediate to upperintermediate levels according to the test performance of the language courses. They had not received any former experience of translation on the day of the study.

Table 1: Demographic information of participants $(\mathrm{N}=44)$

\begin{tabular}{lcr}
\hline Gender & Frequency & \multicolumn{2}{c}{ Percentage } \\
\hline Male & 19 & 43.2 \\
\hline Female & 25 & 56.8 \\
\hline
\end{tabular}

\subsection{Method}

A mixed method was applied in this study to investigate both subjective and objective variables from the participants' perspective to collect a thorough set of data for further analysis (Creswell, 2017)

\subsection{Instrument}

Questionnaire: A questionnaire of eighteen close questions and five open-ended questions was delivered to the participants. The questionnaire consists of three sections including (1) participants' demographic information; (2) learners' attitude to words the target text assessment features; and (3) learners' further concerns in self-assessment. The five-point Likert scale was adopted to investigate the intensity of difficulties English majors encountered in self-evaluation of translation quality from English to Vietnam. The items range from 1-None; 2-Very mild; 3-Mild; 4-Moderate; and 5-Severe. The interpretation of the average value varies from 1 to 5 with the interval scale of 0.8 . The questionnaire was adapted from Newmark's Framework (1988) and Munday's functional framework (2015) of translation quality assessment. In addition, five open-ended questions based on the statement of the problem were integrated to enhance the quality and completeness of the data (Couper et al., 2001). 


\subsection{Data collection and analysis}

An online questionnaire was delivered to fifty target participants of English majors in translation and interpretation at the Faculty of Foreign Languages, Nguyen Tat Thanh University. However, after two weeks of the survey, only forty seven samples were returned (94\%) and forty four samples (88\%) were used for further analysis. An SPSS version 20 tool was used to analyse the variables of the data collection for the mean scores, standard deviation, and frequency.

\section{Results and discussion}

4.1 Learners' attitude towards the difficulties in self-assessment of English- Vietnamese translation quality

\begin{tabular}{|c|c|c|c|}
\hline No. & Difficulties & Mean & STD \\
\hline 1 & Culture & 3.82 & 0.85 \\
\hline 2 & Readership & 3.45 & 0.96 \\
\hline 3 & Setting & 3.64 & 1.05 \\
\hline 4 & Translators' personality & 2.82 & 1.14 \\
\hline 5 & Grammar & 3.73 & 0.94 \\
\hline 6 & Vocabulary & 3.64 & 0.79 \\
\hline 7 & Terminology & 3.95 & 0.95 \\
\hline 8 & Figure of speech & 3.18 & 0.91 \\
\hline 9 & Referentiality & 3.27 & 0.98 \\
\hline 10 & Field & 3.55 & 0.96 \\
\hline 11 & Function & 3.73 & 1.12 \\
\hline 12 & Writing style & 3.45 & 0.91 \\
\hline 13 & Mode & 3.64 & 0.95 \\
\hline 14 & Tenor & 3.59 & 0.96 \\
\hline 15 & Coherence & 3.73 & 0.83 \\
\hline 16 & Cohesion & 3.82 & 1.01 \\
\hline 17 & Naturalness & 4.00 & 0.82 \\
\hline 18 & Texture & 3.36 & 0.73 \\
\hline
\end{tabular}

Figure 3: Learners' attitude towards the intensity of difficulties in self-evaluation of translation quality assessment

The figure 3 shows the attitudes of English majors in translation and interpretation towards the intensity of difficulties they encounter during self-assessment in the stage of translation quality assessment. In general, all of the items received the opinions from the mild degree of difficulty to the moderate degree in assessing their personal translation products. Meanwhile, the standard deviation ranges from 0.73 to 1.14 , which is approximately equivalent to one class in the interval scale. The majority of students had difficulties in determining the level of naturalness which was in the highest mean score at 4.00 while translators' personality was the least at 2.82. In addition to translators' personality, referentiality, figure of speech, and texture were all in the mild degree of difficulties the student translators had to deal with at 3.18, 3.27, and 3.36 respectively. On the other hand, other exploratory items were in the moderate degree. It is worth pointing out that culture and coherence had the same mean score at 3.82. This case is identical for grammar, function, and coherence at 3.73. 
In other words, most of the participants had to confront the challenges from language proficiency in both the source language and the target language. This result is correlated to the idea of Baker (2018) and Nguyen \& Nguyen (2019) about learners' difficulties in translation assessment. In particular, learners had difficulties in working out the exact equivalence for the word level when translating the text from English into Vietnamese. Some words are more or less general than the intended meaning of the expressions. The lack of insights into Vietnamese prevented learners from determining whether the translated words could be acceptable or not in Vietnamese cultures. Considering the grammatical structures between English and Vietnamese, learners experienced obstacles due to the long term exposure to English which reflects and affect their cognition of Vietnamese grammar. Hence, their translation was sometimes influenced by English grammar such as passive voice or tenses. Also, the elicitation of cohesive devices between two languages still caused problems for students to assess the acceptability of the expressions to opt for the direct or indirect speech acts.

In terms of functionalism, learners were only able to convey the meaning of the text from the surface of English with little regards to the in-depth meaning to fully perform the functional expression in Vietnamese. Three components of register including field, tenor, and mode were still challenging as learners did not have enough time and background knowledge to relate the text to the target participants of the speech community in the original text. This idea is also suggested by Munday (2016) and Katan (2018). The time constraint did not allow learners to successfully assess the intentions of utterances in the text to fulfil the desire of expression and generate the same effects for the learners in the target context.

Moreover, as learners dealt with the separate text with no previous or following materials, which breaks the textuality among parts of the whole materials, learners struggled to refer to the external and authentic situation and setting of the text. This level required the investment of time and effort in text analysis to comprehend the figures of speech in the original expression (House, 2015). In order to achieve the level of naturalness in translation, learners need to become a master of the target language but they were not able to assess this level as they experienced the formal tertiary education with English as a medium of instruction. Additionally, naturalness cannot be understood independently from culture. The difficulties of assessing the appropriateness in cultures stemmed from the lack of exposure to both English and Vietnamese. This idea demands for real-world interactions to sense the practicality and authenticity of the texture.

\subsection{Learners' concerns of the assessment framework for translation quality}

The information from the open questions to explore further difficulties in self-evaluation of translation quality in the postprocess indicated the attention of learners to the official rubric for English - Vietnamese translation.

“I am not sure I am right or wrong" (Student 6)

"I dont know how to assess my translation" (Student 14)

This reality resulted in the subjectivity of translation with no exact criteria to check the applicability of translation works. They regularly adopt personal intuition to check the quality of their translation with little reference to the standardized scale.

"I think I use most of my feelings because I just know it is right but I don't know why" (Student 31)

"I don't know the standards to check" (Student 40)

In addition, the absence of translation framework caused learners' dependence on teachers' perspective in translation quality assessment.

"Teachers ask us to change the words but I dont know why I have to do so" (Student 37)

"We feel ok if the lecturer say our translation is ok" (Student 22)

4.3 The lack of teachers' immediate support for feedback in self-assessment

During the classroom activities, teachers could not provide every learner the support to help them enhance their checking the quality of translation. The teachers stayed focused on some students during the whole lesson.

"No experience of teacher support" (Student 13)

"Teacher just suggest her translation for the class with no explanation" (Student 40)

"only $30 \%$ of students received support" (Student 4) 
The lack of immediate support for particular learners also caused negative attitude towards the teaching activities.

"Not really enthusiastic" (Student 20)

"She dont like me so she doesnt help" (Student 30)

"We have to wait so so long to ask teachers" (Student 25)

This fact may come from the class size of fifty five students which prevent the teachers to provide immediate support for all learners. Hence, this idea calls for the solution from the policy makers to reduce the size as translation is affected by the attitude of the translators to produce the style of the translation in the target culture (Bittner, 2020).

\section{Conclusion}

Although the theme of the study is not new, the results of the study are valuable to the context of teaching translation. Firstly, the teaching and learning of translation must be accompanied by the support of teaching all English and Vietnamese skills in a communicative way. Approaching translation major without adequate language competence in both mother tongue and the target language probably turns the translation effort into failure as difficulties in grammar, vocabulary or reading and writing may inhibit the process of decoding meaning in one language and encoding it in a different language. Therefore, the prerequisite for any translation course should include the upper-intermediate level of language competence. Secondly, the sociolinguistic competence should not be undervalued as rules and norms of interacting are governed by the speech communities the language users participate in. A profound understanding of the similarities and differences of aspects related to culture, speaking conventions, writing styles and discourse will definitely facilitate translation-majored students. The reasonable explanation is that such knowledge not only help enhance students' communication but also clarify to them why the oral or written texts might carry meanings which are not always equivalent in two different languages. Last but not least, the support from instructors is of ultimate importance to make students aware of their strengths and weaknesses, thereby adjusting their translation performance accordingly. The feedback must be immediate and contributive to help students solve the problem they have with their tasks. Postponing or skipping quality feedback might confuse the students and cause dissatisfaction or even resentment against the subject. Importantly, an official framework for translation quality assessment need to be provided to serve as an objective tool for students to monitor their own translation progress. This is essential for student self-study, self-assessment, and peer assessment. Additionally, with the framework at hand, the students are able to focus on the aspects of translation which ensures and enhances their performance.

Since the study has a narrow scope with a small number of participants, its findings do not aim to be generalized to all students in translation courses. However, the implications from this study contribute significantly to curriculum design and development, especially in Nguyen Tat Thanh university, where adjustment to requirements of the subjects as well as the content need to be made. Moreover, the implications contribute ideas for utilizing teaching materials, organizing class activities, giving feedback, and conducting assessment and evaluation. The study also supplies supplementary data related to the translation research for meta-analysis to gain more significant results into the issue. It is recommended that further research should be conducted to investigate the teachers' and other stakeholders' perspectives towards learners' selfevaluation on translation quality assessment to formulate the thorough analysis in English Vietnamese translation quality assessment.

\section{About the authors}

Tri Minh Nguyen is currently a Master candidate in TESOL at Edith Cowan University, Australia. He is also a visiting lecturer at Nguyen Tat Thanh University and an academic director at The $\mathrm{V}$ Language. His research interests cover translation, intercultural communication, and discourse analysis.

Trang Xuan Phuong Dao is currently an English Lecturer in HCMC University of Education, the Founder and General Manager of ESTAR Language Center, and a Teacher Trainer of Dai Truong Phat Corporation. Trang holds a Doctorate in Business Administration and a Master's degree in TESOL. Her fields of interest include business research, applied linguistics and teaching methodology. 


\section{References}

[1] Al-Emara, F. S. M. (2016). Is self-assessment a credible indicator of translation competence. Arab World English Journal, 5, $232-248$.

[2] Baker, M. (2018). In other words: A coursebook on translation (3 ${ }^{\text {rd }}$ Ed.). Routledge.

[3] Beiranvand, M., \& Golandouz, G.R. (2017). The comparative effects of self-assessment and peer feedback on improving translation quality. Journal of Language and Translation, 7, 65-78.

[4] Bittner, H. (2020). Evaluating the evaluator: A novel perspective on translation quality assessment. Routledge.

[5] Creswell, J. W., \& Clark, V. L. P. (2017). Designing and conducting mixed methods research. SAGE.

[6] Creswell, J. W. (2009). Research design: Qualitative, quantitative, and mixed methods approaches (3rd ed.). SAG.

[7] Colina, S. (2015). Fundamentals of translation. Cambridge University Press.

[8] Han, C., \& Riazi, M. (2018). The accuracy of student self-assessments of English-Chinese bidirectional interpretation: A longitudinal quantitative study. Assessment \& Evaluation in Higher Education, 1-13. doi:10.1080/02602938.2017.1353062

[9] House, J. (2015). Translation quality assessment: Past and present. Routledge.

[10] Huertas-Barros, E., Vandepitte, S., \& Iglesias-Fernandez, E. (2019). Quality assurance and assessment practices in translation and interpreting. IGI Global.

[11] Katan, D. (2014). Translating cultures: An introduction for translators, interpreters, and mediators. Routledge.

[12] Kenny, D. (2009). Equivalence. In Mona Baker and Gabriela Saldanha (Eds) Routledge Encyclopedia of Translation Studies (2nd ed., pp. 96-99). Routledge.

[13] Lee, Y-H. (2005). Self-assessment as an autonomous learning tool in an interpretation classroom. Meta, 50(4). doi:10.7202/019869ar

[14] Maitland, S. (2017). What is cultural translation?. Bloomsbury.

[15] Munday, J. (2016). Introducing translation studies: Theories and applications (4 ${ }^{\text {th }}$ Ed.). Routledge.

[16] Newmark, P. (1988). A textbook of translation. Prentice Hall International.

[17] Nguyen, T. K. T., \& Nguyen, L. U. M. (2019). Difficulties in translating TV programs in English into Vietnamese: A study at Lam Dong radio-television station. International Journal on Studies in English Language and Literature, 7 (5), 13-26.

[18] Nida, E. (2002). Contexts in translating. John Benjamins.

[19] Pym, A. (2010). Exploring translation theories. Routledge.

[20] Pym, A. (2017). Natural and directional equivalence in theories of translation. Target, 19 (2), 271-294.

[21] Sandrelli, A. (2015). Becoming an interpreter: The role of computer technology. MonTI, 2, 111-138.

[22] Sathisha, M. (2020). Linguistic and cultural challenges faced by translators. International Journal of Psychological Rehabilitation, 24 (2), 178-182.

[23] Witter-Merithew, A., Taylor, M., \& Johnson, L. (2001). Guided self-assessment and professional development planning: A model applied to interpreters in educational settings. In C. Nettles (Ed.), Proceedings of the 17th national conference of the registry of interpreters for the deaf (pp. 153-226). RID Publications.

\section{QUESTIONNAIRE}

\section{AN INVESTIGATION INTO LEARNERS' DIFFICULTIES IN SELF-EVALUATION IN ENGLISH- VIETNAMESE TRANSLATION QUALITY ASSESSMENT}

\section{Section 1: Participants' information}

1. What is your full name?

2. What is your email address?

3. What is your student ID?

4. What is your gender?

A. Male

B. Female

5. Do you have previous experience in translation?

A. Yes

B. No 


\section{Section 2: The investigation}

2.1 In the scale from 1 to 5, please indicate how much your experience the difficulties in self-evaluation for the following features in translation quality assessment from English to Vietnamese

1. None

2. Very mild

3. Mild

4. Moderate

5. Severe

\begin{tabular}{|c|c|c|c|c|c|c|}
\hline No. & Difficulties & 1 & 2 & 3 & 4 & 5 \\
\hline 1 & Culture & & & & & \\
\hline 2 & Readership & & & & & \\
\hline 3 & Setting & & & & & \\
\hline 4 & Translators' personality & & & & & \\
\hline 5 & Grammar & & & & & \\
\hline 6 & Vocabulary & & & & & \\
\hline 7 & Terminology & & & & & \\
\hline 8 & Figure of speech & & & & & \\
\hline 9 & Referentiality & & & & & \\
\hline 10 & Field & & & & & \\
\hline 11 & Function & & & & & \\
\hline 12 & Writing style & & & & & \\
\hline 13 & Mode & & & & & \\
\hline 14 & Tenor & & & & & \\
\hline 15 & Coherence & & & & & \\
\hline 16 & Cohesion & & & & & \\
\hline 17 & Naturalness & & & & & \\
\hline 18 & Texture & & & & & \\
\hline
\end{tabular}

2.2 What are others difficulties you encounter in assessing the quality of your translation from English into Vietnamese?

2.3 How do your teachers assist you in checking the quality of your translation? 